\title{
An Improved Finite-Time Stability and Stabilization of Linear System with Constant Delay
}

\author{
Thaned Rojsiraphisal and Jirapong Puangmalai \\ Department of Mathematics, Faculty of Science, Chiang Mai University, Chiang Mai 50200, Thailand \\ Correspondence should be addressed to Thaned Rojsiraphisal; thaned.r@cmu.ac.th
}

Received 21 August 2014; Revised 15 December 2014; Accepted 16 December 2014; Published 28 December 2014

Academic Editor: Vu Ngoc Phat

Copyright ( 2014 T. Rojsiraphisal and J. Puangmalai. This is an open access article distributed under the Creative Commons Attribution License, which permits unrestricted use, distribution, and reproduction in any medium, provided the original work is properly cited.

\begin{abstract}
Practical systems in engineering fields often require that values of state variables, during the finite-time interval, must not exceed a certain value when the initial values of state are given. This leads us to investigate the finite-time stability and stabilization of a linear system with a constant time-delay. Sufficient conditions to guarantee the finite-time stability and stabilization are derived by using a new form of Lyapunov-Krasovskii functional and a desired state-feedback controller. These conditions are in the form of LMIs and inequalities. Two numerical examples are given to show the effectiveness of the proposed criteria. Results show that our proposed criteria are less conservative than previous works in terms of versatility of minimum bounds and larger bounds of time-delay.
\end{abstract}

\section{Introduction}

In the past decades, researchers have paid much attention to asymptotic stability which concerns behaviors of state variables over an infinite time interval. One disadvantage of the asymptotic stability behavior is that large values of state variables may present during transient period. In practical system, the presence of large values should not exceed its limit, for example, the presence of saturations or the excitation of nonlinear dynamics $[1,2]$. This leads us to a concept called finite-time stability, introduced back in 1960s. This concept is focusing on the behavior of state variables during the transient period which must not exceed a certain value when the upper bound of initial condition is given (see [1, 3-5]). Researchers have studied finite-time stability on various systems such as linear system, impulsive system, neural networks, and switched systems (see [1-18] and references therein) and proposed sufficient conditions to guarantee finite-time stability in the forms of linear matrix inequality (LMI), Lyapunov differential matrix equation, or algebraic inequality, and so forth.

Time-delay often occurs in practical systems such as biological systems, chemical systems, electrical networks, and engineering fields. It is known that the small change of timedelay can cause instability and poor performance of such systems. Considering the broad applications related to the time-delay and the suitable values of state variables during the transient, it is important to investigate the finite-time stability and stabilization of systems with time-delay. From literature, the studies of finite-time stability and finite-time stabilization on time-delay system are not many $[9,12-16,18-21]$ and only few studies are on linear system with time-delay $[9,11,16]$.

In this paper, we propose new sufficient conditions on finite-time stability and stabilization for a linear system with constant time-delay in the form of LMIs. The proposed conditions are formulated using a new form LyapunovKrasovskii functional. To illustrate the efficiency of the proposed conditions, two numerical examples are presented at the end.

\section{Preliminaries}

The following notations will be used in this paper. $R^{n}$ denotes the $n$-dimensional space with the scalar product $x^{T} y$ and the vector norm $\|\cdot\| ; R^{n \times n}$ denotes an $n \times n$ matrix with real value elements; $A^{T}$ denotes the transpose of the matrix $A ; \lambda(A)$ 
denotes eigenvalues of $A ; \lambda_{\max }(A)$ and $\lambda_{\min }(A)$ represent the maximum and minimum of real part of $\lambda(A)$, respectively. $x_{t}:=\{x(t+s): s \in[-\tau, 0]\} ;\left\|x_{t}\right\|:=\sup _{s \in[-\tau, 0]}\{\|x(t+s)\|, \| \dot{x}(t+$ $s) \|\} ; A>0(A<0)$ means $A$ is positive (negative) definite; $A>B$ is equivalent to $A-B>0$. Entries $*$ in a matrix represent the symmetric elements of the symmetric matrix.

Consider a linear system with constant time-delay

$$
\dot{x}(t)=A_{0} x(t)+A_{1} x(t-\tau)+B u(t)
$$

where $t>0$ and $x(t) \in R^{n}$ is the state vector of the system. $u(t) \in R^{m}$ is the control input. $A_{0}, A_{1} \in R^{n \times n}$ and $B \in$ $R^{n \times m}$ are known constant matrices. The delay $\tau$ is a positive constant.

We assume that the initial condition is $x(t+\theta)=\phi(\theta)$, $\forall \theta \in[-\tau, 0]$, and $\phi(\cdot)$ is a differentiable vector-valued initial function with the norm $\|\phi\|:=\sup _{t \in[-\tau, 0]}\{\|\phi(t)\|,\|\dot{\phi}(t)\|\}$.

Here we choose a state-feedback control law of the form

$$
u(t)=-0.5 B^{T} P x(t)
$$

where $P$ is a design parameter to be determined.

To formulate sufficient conditions of finite-time stability and stabilization of the linear system (1), the following definition and lemmas are used.

Definition 1. System (1) is said to be finite-time stable with respect to $\left(c_{1}, c_{2}, T\right)$, where $0 \leq c_{1}<c_{2}$, if

$$
\|\phi\|^{2} \leq c_{1} \Longrightarrow\|x(t)\|^{2} \leq c_{2}, \quad \forall t \in[0, T]
$$

Lemma 2 (see [22]). For any symmetric positive-definite matrix $M \in \mathbb{R}^{n \times n}$, scalar $\sigma \geq 0$, and vector function $w$ : $[0, \sigma] \rightarrow \mathbb{R}^{n}$ such that the concerned integrations are well defined, then

$$
\left(\int_{0}^{\sigma} w(s) d s\right)^{T} M\left(\int_{0}^{\sigma} w(s) d s\right) \leq \sigma \int_{0}^{\sigma} w^{T}(s) M w(s) d s .
$$

Lemma 3 (see [23], Schur complement). Given constant matrices $X, Y, Z$ with appropriate dimensions satisfying $X=$ $X^{T}, Y=Y^{T}>0$, then $X+Z^{T} Y^{-1} Z<0$ if and only if

$$
\left[\begin{array}{cc}
X & Z^{T} \\
Z & -Y
\end{array}\right]<0 \quad \text { or } \quad\left[\begin{array}{cc}
-Y & Z \\
Z^{T} & X
\end{array}\right]<0
$$

\section{Main Results}

In this section, we first formulate the finite-time stability of the linear system (1) without controller, that is, $u(t)=0$. We then provide proof of the finite-time stabilization of the linear system (1) with a feedback controller defined in (2). The formulations are as follows.

Theorem 4. Linear system (1) with $u(t)=0$; time-delay $\tau$ is finite-time stable with respect to $\left(c_{1}, c_{2}, T\right), 0 \leq c_{1}<c_{2}$, if there exist nonnegative scalar $\alpha$, symmetric positive-definite matrices $P, Q, R$, and positive scalars $\beta_{1}, \beta_{2}, \beta_{3}, \beta_{4}$ such that

$$
\begin{gathered}
\beta_{1} I<P<\beta_{2} I, \\
0<Q<\beta_{3} I, \\
0<R<\beta_{4} I, \\
\Omega=\left[\begin{array}{cccc}
-\beta_{1} c_{2} e^{-\alpha T} & \beta_{2} \sqrt{c_{1}} & \beta_{3} \sqrt{c_{1} \tau} & \beta_{4} \sqrt{0.5 c_{1} \tau^{3}} \\
* & -\beta_{2} & 0 & 0 \\
* & * & -\beta_{3} & 0 \\
* & * & * & -\beta_{4}
\end{array}\right]<0, \\
\Psi=\left[\begin{array}{cccc}
\Psi_{11} & P A_{1}+\tau^{2} A_{0}^{T} R A_{1}+R \\
* & \tau^{2} A_{1}^{T} R A_{1}-Q-R
\end{array}\right]<0,
\end{gathered}
$$

where

$$
\Psi_{11}=A_{0}^{T} P+P A_{0}+Q+\tau^{2} A_{0}^{T} R A_{0}-R-\alpha P .
$$

Proof. Let us consider the following Lyapunov-Krasovskii functional:

$$
\begin{aligned}
V(x(t))= & e^{\alpha t} x^{T}(t) P x(t) \\
& +e^{\alpha t} \int_{t-\tau}^{t} x^{T}(s) Q x(s) d s \\
& +\tau e^{\alpha t} \int_{-\tau}^{0} \int_{t+s}^{t} \dot{x}^{T}(\theta) R \dot{x}(\theta) d \theta d s .
\end{aligned}
$$

The derivative of $V(x(t))$ along solution (1) with $u(t)=0$ is

$$
\begin{aligned}
\dot{V}= & 2 e^{\alpha t} x^{T}(t) P \dot{x}(t) \\
& +e^{\alpha t}\left[x^{T}(t) Q x(t)-x^{T}(t-\tau) Q x(t-\tau)\right] \\
& +\tau e^{\alpha t}\left[\tau \dot{x}^{T}(t) R \dot{x}(t)-\int_{t-\tau}^{t} \dot{x}^{T}(s) R \dot{x}(s) d s\right]+\alpha V \\
= & 2 e^{\alpha t} x^{T}(t) P\left[A_{0} x(t)+A_{1} x(t-\tau)\right] \\
& +e^{\alpha t}\left[x^{T}(t) Q x(t)-x^{T}(t-\tau) Q x(t-\tau)\right] \\
& +\tau e^{\alpha t}\left[\tau\left[A_{0} x(t)+A_{1} x(t-\tau)\right]^{T}\right. \\
& \times R\left[A_{0} x(t)+A_{1} x(t-\tau)\right] \\
& \left.\quad-\int_{t-\tau}^{t} \dot{x}^{T}(s) R \dot{x}(s) d s\right]+\alpha V .
\end{aligned}
$$


Applying Lemma 2 and the Newton-Leibniz formula, we obtain

$$
\begin{aligned}
\dot{V} \leq & 2 e^{\alpha t} x^{T}(t) P\left[A_{0} x(t)+A_{1} x(t-\tau)\right] \\
+ & e^{\alpha t}\left[x^{T}(t) Q x(t)-x^{T}(t-\tau) Q x(t-\tau)\right] \\
+ & \tau^{2} e^{\alpha t}\left(\left[A_{0} x(t)+A_{1} x(t-\tau)\right]^{T}\right. \\
& \left.\times R\left[A_{0} x(t)+A_{1} x(t-\tau)\right]\right) \\
- & e^{\alpha t}[x(t)-x(t-\tau)]^{T} \\
& \times R[x(t)-x(t-\tau)]+\alpha V .
\end{aligned}
$$

Since $\alpha>0, P>0$, and $V>0$, thus we have

$$
\begin{aligned}
\dot{V}(x(t))- & 2 \alpha V(x(t)) \\
\leq e^{\alpha t}\left\{x^{T}(t)\right. & \\
\times & {\left[A_{0}^{T} P+P A_{0}+Q+\tau^{2} A_{0}^{T} R A_{0}-R-\alpha P\right] x(t) } \\
& +2 x^{T}(t)\left[P A_{1}+\tau^{2} A_{0}^{T} R A_{1}+R\right] x(t-\tau) \\
& \left.+x^{T}(t-\tau)\left[\tau^{2} A_{1}^{T} R A_{1}-Q-R\right] x(t-\tau)\right\} \\
=\eta^{T}(t) & \Psi \eta(t)
\end{aligned}
$$

where $\eta(t)=[x(t), x(t-\tau)]$.

Because $\Psi<0$, thus inequality (13) leads to $\dot{V}(x(t))-$ $2 \alpha V(x(t))<0$. Multiplying this inequality by $e^{-2 \alpha t}$ and integrating from 0 to $t$, with $t \in[0, T]$, we obtain

$$
e^{\alpha t} \lambda_{\min }(P)\|x(t)\|^{2} \leq V(x(t))<e^{2 \alpha t} V(x(0)),
$$

with

$$
\begin{aligned}
V(x(0))= & x^{T}(0) P x(0) \\
& +\int_{-\tau}^{0} x^{T}(s) Q x(s) d s \\
& +\tau \int_{-\tau}^{0} \int_{s}^{0} \dot{x}^{T}(\theta) R \dot{x}(\theta) d \theta d s \\
\leq & \left\{\lambda_{\max }(P)+\tau \lambda_{\max }(Q)+0.5 \tau^{3} \lambda_{\max }(R)\right\}\|\phi\|^{2} .
\end{aligned}
$$

Now we define

$$
\Pi: \equiv c_{1}\left\{\beta_{2}+\tau \beta_{3}+0.5 \tau^{3} \beta_{4}\right\}-c_{2} \beta_{1} e^{-\alpha T}<0,
$$

where $\beta_{1}, \beta_{2}, \beta_{3}, \beta_{4}$ are positive and satisfy (6). Applying Schur compliment, it is easy to see that $\Pi<0$ is equivalent to $\Omega<0$. Because $\|\phi\|^{2} \leq c_{1}$, thus the relations (14)-(15) lead to

$$
\|x(t)\|^{2} \leq \frac{c_{1} e^{\alpha T}}{\lambda_{\min }(P)}\left\{\lambda_{\max }(P)+\tau \lambda_{\max }(Q)+0.5 \tau^{3} \lambda_{\max }(R)\right\} .
$$

With $P, Q, R$ satisfying (6), we have

$$
\|x(t)\|^{2} \leq \frac{c_{1} e^{\alpha T}}{\beta_{1}}\left\{\beta_{2}+\tau \beta_{3}+0.5 \tau^{3} \beta_{4}\right\}<c_{2} .
$$

The proof is complete.

Next consider the linear system (1) with a feedback controller of the form $u(t)=-0.5 B^{T} P x(t)$ as defined in (2). This equation can be rewritten as

$$
\dot{x}(t)=\widehat{A}_{0} x(t)+A_{1} x(t-\tau),
$$

where $\widehat{A}_{0}=A_{0}-0.5 B B^{T} P$.

Remark 5. One can notice that the finite-time stabilization of (1) with the feedback controller above is equivalent to finitetime stability of (19). Thus we can formulate the finite-time stabilization as in the following theorem.

Theorem 6. The linear system (1) with feedback controller (2) is finite-time stabilization with respect to $\left(c_{1}, c_{2}, T\right), 0 \leq c_{1}<$ $c_{2}$, if there exist a scalar $\alpha>0$, symmetric positive-definite matrices $X, Y, Z, R, W_{1}, W_{2}$, and matrices $W_{3}, W_{4}, W_{5}$ such that

$$
\Sigma=\left[\begin{array}{cc}
\Sigma_{11} & \Sigma_{12} \\
* & \Sigma_{22}
\end{array}\right]<0,
$$

where

$$
\begin{gathered}
\Sigma_{11}=X A_{0}^{T}+A_{0} X+Y-Z+\tau^{2} W_{1}-\tau^{2} B B^{T} W_{4} \\
+0.25 \tau^{2} B B^{T} R B B^{T}-B B^{T}-\alpha X, \\
\Sigma_{12}=A_{1} X+Z+\tau^{2} W_{3}-0.5 \tau^{2} B B^{T} W_{5}, \\
\Sigma_{22}=\tau^{2} W_{2}-Y-Z, \\
c_{1} e^{\alpha T} \lambda_{\max }(X)\left\{\frac{1}{\lambda_{\min }(X)}+\frac{\tau}{\lambda_{\min }\left(X Y^{-1} X\right)}\right. \\
\left.+\frac{0.5 \tau^{3}}{\lambda_{\min }\left(X Z^{-1} X\right)}\right\}<c_{2} .
\end{gathered}
$$

Proof. Replacing $A_{0}$ in the LMI (8) with $\widehat{A}_{0}=A_{0}-0.5 B B^{T} P$, we obtain

$$
\widehat{\Psi}=\left[\begin{array}{cc}
\widehat{\Psi}_{11} & \widehat{\Psi}_{12} \\
* & \widehat{\Psi}_{22}
\end{array}\right]<0,
$$

where

$$
\begin{gathered}
\widehat{\Psi}_{11}=A_{0}^{T} P+P A_{0}+Q+\tau^{2} A_{0}^{T} R A_{0}-R-\alpha P \\
-P B B^{T} P+0.25 \tau^{2} P B B^{T} R B B^{T} P-\tau^{2} P B B^{T} R A_{0} \\
\widehat{\Psi}_{12}=P A_{1}+R+\tau^{2} A_{0}^{T} R A_{1}-0.5 \tau^{2} P B B^{T} R A_{1}, \\
\widehat{\Psi}_{22}=\tau^{2} A_{1}^{T} R A_{1}-Q-R .
\end{gathered}
$$




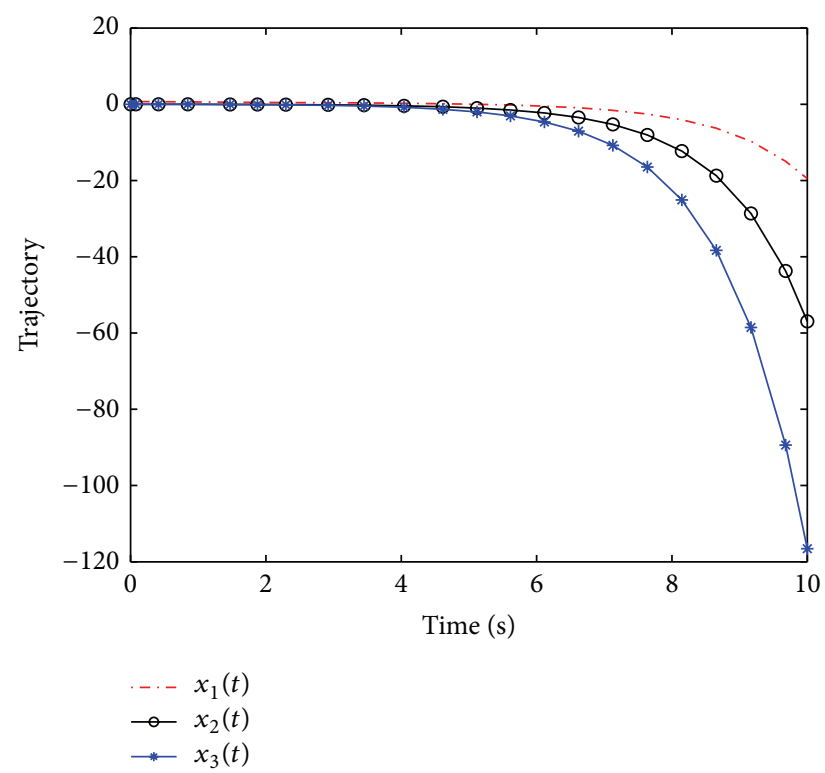

(a)

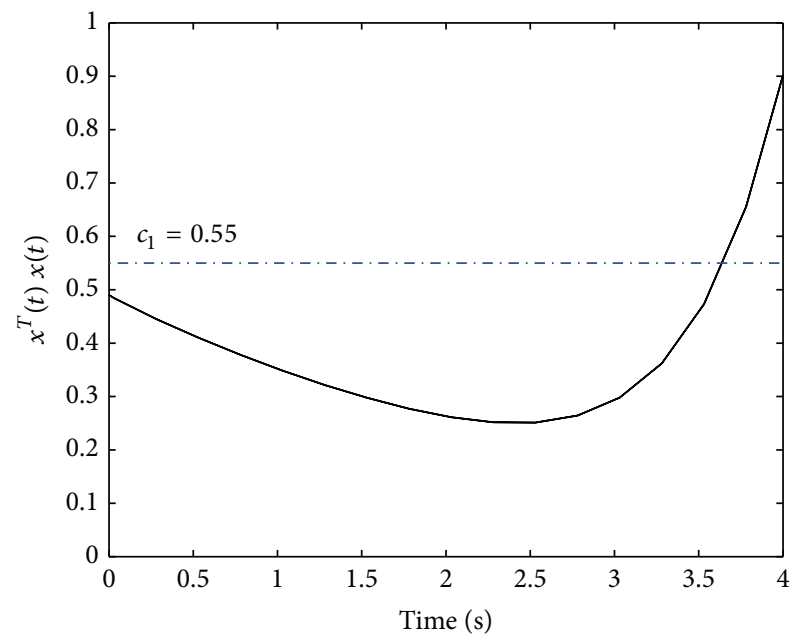

(b)

FIGURE 1: State trajectories (a) and norm of the state trajectories (b) of the linear system (28) with initial condition $\phi^{T}(t)=[0.7,0,0]$.

Pre- and postmultiplying the above inequality by $\operatorname{diag}\left\{P^{-1}, P^{-1}\right\}$, we obtain

$$
\left[\begin{array}{cc}
\widehat{\Sigma}_{11} & \widehat{\Sigma}_{12} \\
* & \widehat{\Sigma}_{22}
\end{array}\right]<0
$$

where

$$
\begin{aligned}
\widehat{\Sigma}_{11}= & P^{-1} A_{0}^{T}+A_{0} P^{-1}+P^{-1} Q P^{-1} \\
& +\tau^{2} P^{-1} A_{0}^{T} R A_{0} P^{-1}-P^{-1} R P^{-1}-\alpha P^{-1} \\
& -B B^{T}-\tau^{2} B B^{T} R A_{0} P^{-1}+0.25 \tau^{2} B B^{T} R B B^{T}, \\
\widehat{\Sigma}_{12}= & A_{1} P^{-1}+P^{-1} R P^{-1}+\tau^{2} P^{-1} A_{0}^{T} R A_{1} P^{-1} \\
& \quad-0.5 \tau^{2} B B^{T} R A_{1} P^{-1}, \\
\widehat{\Sigma}_{22}= & \tau^{2} P^{-1} A_{1}^{T} R A_{1} P^{-1}-P^{-1} Q P^{-1}-P^{-1} R P^{-1} .
\end{aligned}
$$

Set $X=P^{-1}, Y=P^{-1} Q P^{-1}, Z=P^{-1} R P^{-1}, W_{1}=$ $P^{-1} A_{0}^{T} R A_{0} P^{-1}, W_{2}=P^{-1} A_{1}^{T} R A_{1} P^{-1}, W_{3}=P^{-1} A_{0}^{T} R A_{1} P^{-1}$, $W_{4}=R A_{0} P^{-1}$, and $W_{5}=R A_{1} P^{-1}$. Thus, LMI (25) is equivalent to (20).

With the setting relations above, inequalities (17) in Theorem 4 can be bounded by

$$
\begin{aligned}
& \|x(t)\|^{2} \\
& \quad \leq \frac{c_{1} e^{\alpha T}}{\lambda_{\min }(P)}\left\{\lambda_{\max }(P)+\tau \lambda_{\max }(Q)+0.5 \tau^{3} \lambda_{\max }(R)\right\}
\end{aligned}
$$

$$
\begin{gathered}
=c_{1} e^{\alpha T} \lambda_{\max }(X)\left\{\frac{1}{\lambda_{\min }(X)}+\frac{\tau}{\lambda_{\min }\left(X Y^{-1} X\right)}\right. \\
\left.+\frac{0.5 \tau^{3}}{\lambda_{\min }\left(X Z^{-1} X\right)}\right\}<c_{2} .
\end{gathered}
$$

The proof is complete.

\section{Numerical Examples}

In this section, we give two numerical examples to show the effectiveness of our main results by investigating the linear system of the form

$$
\dot{x}(t)=A_{0} x(t)+A_{1} x(t-\tau)+B u(t),
$$

where

$$
\begin{array}{cc}
A_{0}=\left[\begin{array}{ccc}
-1.7 & 1.7 & 0 \\
1.3 & -1 & 0.7 \\
0.7 & 1 & -0.6
\end{array}\right], \quad A_{1}=\left[\begin{array}{ccc}
1.5 & -1.7 & 0.1 \\
-1.3 & 1 & -0.3 \\
-0.7 & 1 & 0.6
\end{array}\right], \\
B=\left[\begin{array}{l}
-1 \\
10 \\
20
\end{array}\right], & \tau=0.2 .
\end{array}
$$

Remark 7. The linear system (28) with $u(t)=0$ is not asymptotically stable with initial condition $\phi^{T}(t)=[0.7,0,0]$ as shown in Figure 1. The figure reveals that the state variables $x_{i}(t) \rightarrow \infty, i=1,2,3$, as $t \rightarrow \infty$. In the next example we will show that this system is finite-time stable. 


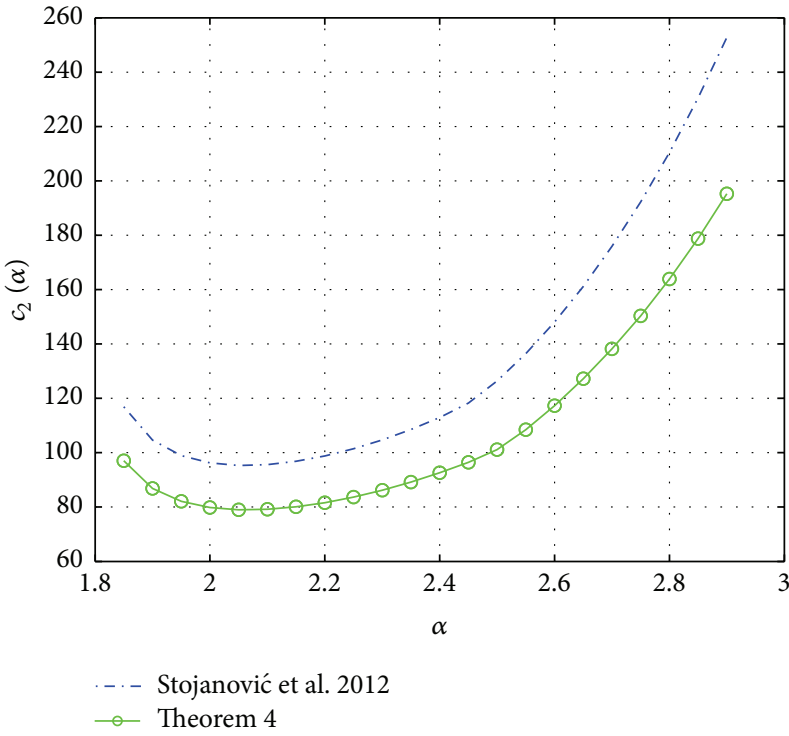

(a)

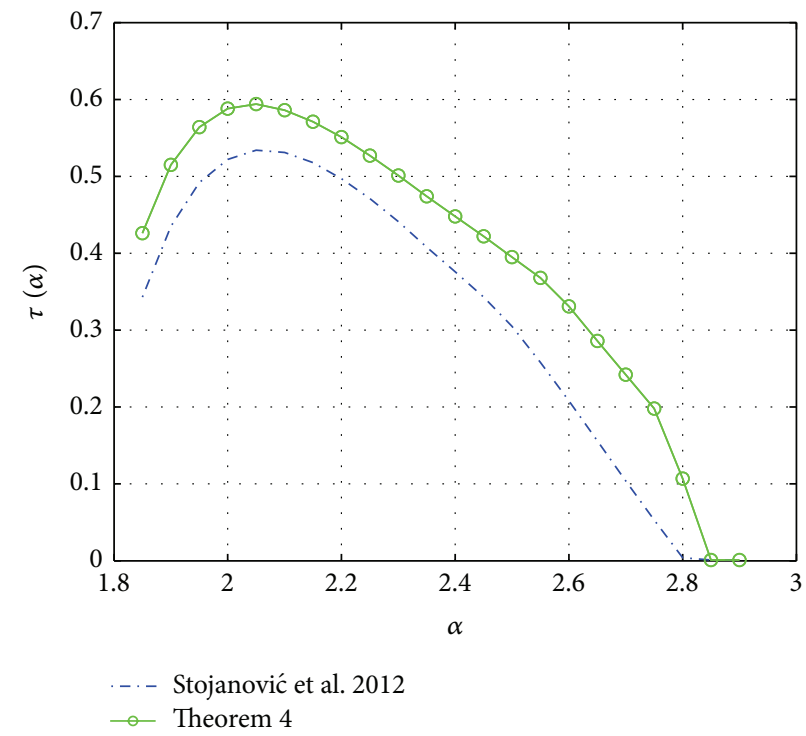

(b)

Figure 2: Comparing the values of $c_{2}$ (a) and the time-delay $\tau$ (b) when $\alpha$ is varied guaranteeing FTS of the linear system (28).

Example 1. Consider the finite-time stability of linear system (28) with respect to $\left(c_{1}, c_{2}, T\right)=(0.55,100,2)$ and $\phi^{T}(t)=$ $[0.7,0,0]$ without the controller; that is, $u(t)=0$. Note that $\phi^{T}(t) \phi(t)=0.49<0.55=c_{1}$. For fixed $\alpha=1.95$, we solve inequalities (6) and LMIs (7) and (8) using MATLAB control toolbox. The feasible solutions guaranteeing finitetime stability of the linear system (28) are $\beta_{1}=4.5462$, $\beta_{2}=11.1083, \beta_{3}=26.8644, \beta_{4}=18.9680$, and

$$
\begin{aligned}
P & =\left[\begin{array}{ccc}
7.1646 & 0.3981 & 0.0289 \\
0.3981 & 10.8567 & -0.5974 \\
0.0289 & -0.5974 & 4.6536
\end{array}\right], \\
Q & =\left[\begin{array}{ccc}
18.1534 & -7.8255 & -4.4657 \\
-7.8255 & 18.5720 & -2.9397 \\
-4.4657 & -2.9397 & 2.8834
\end{array}\right], \\
R & =\left[\begin{array}{ccc}
12.4769 & -1.6625 & 2.1735 \\
-1.6625 & 6.3204 & -2.6576 \\
2.1735 & -2.6576 & 1.8071
\end{array}\right] .
\end{aligned}
$$

We further investigate the finite-time stability of the linear system by comparing the smallest eligible value of $c_{2}$ and the largest eligible value of delay $\tau$ when $\alpha$ varies between condition given in [16] and our condition in Theorem 4. Comparing results are plotted in Figure 2. From this figure, one can clearly see that our condition allows smaller value of $c_{2}$ (Figure 2(a)) and larger value of delay $\tau$ (Figure 2(b)) for all values of $\alpha$. Moreover, we observe that the optimal values of $c_{2}$ and $\tau$ are obtained when $\alpha \in[2.05,2.1]$. For $\alpha=2.05$ is fixed, Theorem 4 allows smaller value of $c_{2}$ by $17.1 \%$ and larger value of $\tau$ by $11.2 \%$.

In addition, we compare the smallest eligible value of $c_{2}$ for two different time-delays $(\tau=0.2,0.5)$ with $\alpha=2.1$. Results, as shown in Table 1, reveal that Theorem 4 allows smallest value of $c_{2}$ compared with the others. Our lower
TABLE 1: The smallest value of $c_{2}$ for $c_{1}=0.55, T=2$, and $\alpha=2.1$.

\begin{tabular}{lcc}
\hline$\tau$ & 0.2 & 0.5 \\
\hline$[4]($ Theorem 6) & $1.6 \times 10^{5}$ & $2.01 \times 10^{5}$ \\
{$[12]($ Theorem 3) } & $2.46 \times 10^{10}$ & $2.46 \times 10^{10}$ \\
{$[16]$ (Corollary 1) } & 95.59 & 145.04 \\
Theorem 4 & 79.18 & 135.08 \\
\hline
\end{tabular}

bounds of $c_{2}$ are smaller than those given in [16] by $17.1 \%$ and $6.8 \%$ for $\tau=0.2,0.5$, respectively. In other words, our proposed condition for finite-time stability of the linear system (28) would be more tolerant for smaller values of $c_{2}$ than conditions given in $[4,12,16]$. Note that results obtained in $[4,12]$ do not use LMI technique.

Example 2. Consider the finite-time stabilization of linear system (28) with a nonzero feedback controller defined in (2) with respect to $\left(c_{1}, c_{2}, T\right)=(0.55,5,10)$. Solving LMI $(20)$ and inequality (22), for $\alpha=1.1 \times 10^{-5}$, we obtain the feasible solutions as follows:

$$
\begin{aligned}
& X=\left[\begin{array}{ccc}
26.3675 & 12.3498 & -5.8926 \\
12.3498 & 22.4784 & 5.2751 \\
-5.8926 & 5.2751 & 34.4024
\end{array}\right], \\
& Y=\left[\begin{array}{ccc}
26.0383 & -2.6851 & 1.4135 \\
-2.6851 & 26.0587 & -0.8541 \\
1.4135 & -0.8541 & 25.1168
\end{array}\right], \\
& Z=\left[\begin{array}{ccc}
23.1998 & 2.6851 & -1.4135 \\
2.6851 & 23.1793 & 0.8541 \\
-1.4135 & 0.8541 & 24.1213
\end{array}\right],
\end{aligned}
$$




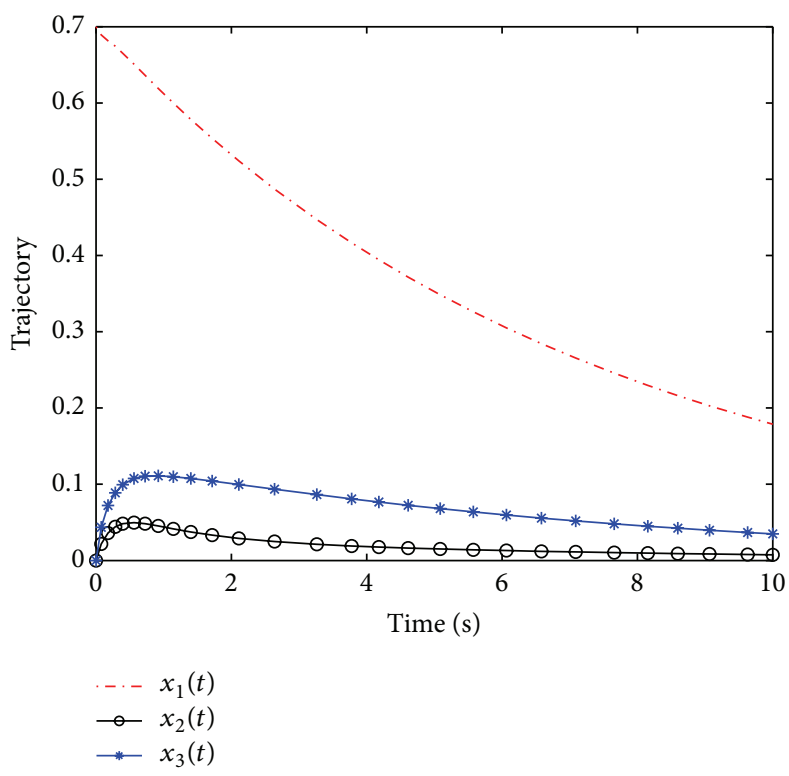

(a)

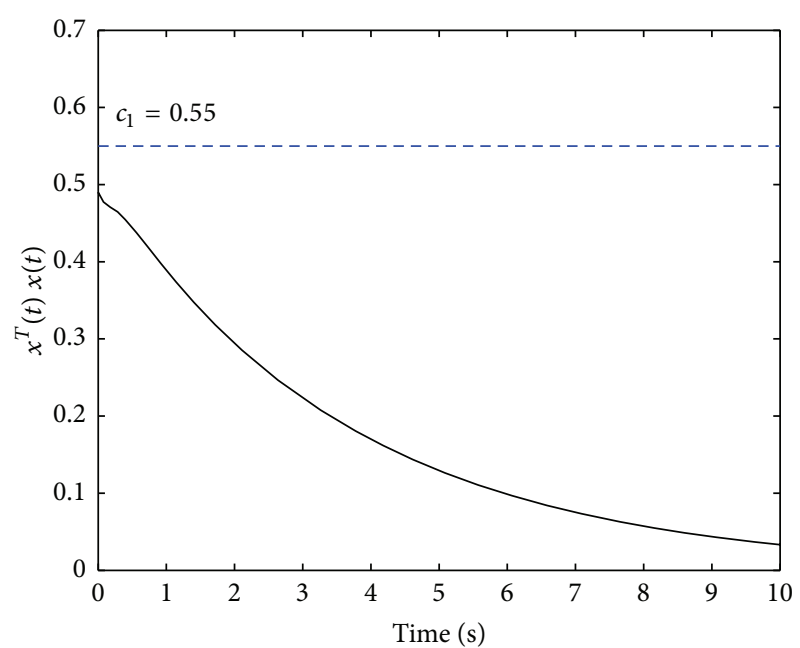

(b)

FiguRE 3: State trajectories (a) and norm of the state trajectories (b) of the linear system (28) with controller $u(t)=[0.0526,-0.1921,-0.2522]$ and initial condition $\phi^{T}(t)=[0.7,0,0]$.

$$
\begin{aligned}
& R=\left[\begin{array}{ccc}
36.1954 & 0.0000 & -0.0000 \\
0.0000 & 36.1954 & 0.0000 \\
-0.0000 & 0.0000 & 36.1954
\end{array}\right], \\
& W_{1}=\left[\begin{array}{ccc}
36.2521 & -0.1074 & 0.0565 \\
-0.1074 & 36.2530 & -0.0342 \\
0.0565 & -0.0342 & 36.2153
\end{array}\right] \text {, } \\
& W_{2}=\left[\begin{array}{ccc}
36.6584 & -0.0000 & 0.0000 \\
-0.0000 & 36.6584 & -0.0000 \\
0.0000 & -0.0000 & 36.6584
\end{array}\right] \text {, } \\
& W_{3}=\left[\begin{array}{ccc}
-996.5122 & 365.9789 & 332.9969 \\
110.2025 & -241.3045 & 528.2569 \\
-376.9375 & 472.0982 & -124.1091
\end{array}\right] \text {, } \\
& W_{4}=10^{3} \times\left[\begin{array}{ccc}
0.0678 & 0.4369 & 3.3416 \\
-0.4318 & 4.3559 & 9.1266 \\
0.0011 & 0.1027 & 0.1123
\end{array}\right] \text {, } \\
& W_{5}=10^{4} \times\left[\begin{array}{ccc}
6.8609 & 7.9208 & 0.0197 \\
0.1372 & 0.1584 & 0.0004 \\
0.2744 & 0.3168 & 0.0008
\end{array}\right] \text {, } \\
& c_{1} e^{\alpha T} \lambda_{\max }(X)\left\{\frac{1}{\lambda_{\min }(X)}+\frac{\tau}{\lambda_{\min }\left(X Y^{-1} X\right)}\right. \\
& \left.+\frac{0.5 \tau^{3}}{\lambda_{\min }\left(X Z^{-1} X\right)}\right\}=3.59<5=c_{2} \text {. }
\end{aligned}
$$

Here, the feedback controller guarantees that finite-time stabilization of the linear system is designed by $u(t)=$ $-0.5 B^{T} X^{-1}=[0.0526,-0.1921,-0.2522]$. Figure 3 shows the trajectories of states (Figure 3(a)) and its norm (Figure 3(b)) with initial condition $\phi^{T}(t)=[0.7,0,0]$. It can be observed that each trajectory is bounded between 0 and 0.7 for all $t \in[0,10]$ and its norm $x^{T}(t) x(t)$ is bounded by $c_{1}=0.55$. In fact, we further extend the time interval and observe that the norm $x^{T}(t) x(t)<0.55$ for all $t>0$, that is, the linear system (28) with the desired controller, is finite-time stabilization with respect to $(0.55,5, T)$ for all $T>0$.

Remark 8. The derivations of the main theorem above are based upon the Lyapunov-Krasovskii approach. Thus, these conditions are not only finite-time stable, but also asymptotically stable. This behavior can be seen when the time domain in Figure 3 is extended.

\section{Conclusion}

In this paper, the finite-time stability and stabilization conditions of the linear system with constant delay are obtained. The sufficient conditions are formulated using a new form of Lyapunov-Krasovskii functional. Results from both examples illustrate that our proposed criteria are less conservative than other existing works.

\section{Conflict of Interests}

The authors declare that there is no conflict of interests regarding the publication of this paper.

\section{Acknowledgment}

This research is supported by Chiang Mai University, Thailand. 


\section{References}

[1] F. Amato, M. Ariola, and C. Cosentino, "Robust finite-time stabilisation of uncertain linear systems," International Journal of Control, vol. 84, no. 12, pp. 2117-2127, 2011.

[2] L. Liu and J. Sun, "Finite-time stabilization of linear systems via impulsive control," International Journal of Control, vol. 81, no. 6, pp. 905-909, 2008.

[3] P. Dorato, "Short time stability in linear time-varying system," in Proceedings of the IRE International Convention Record Part 4, pp. 83-87, New York, NY, USA, 1961.

[4] D. L. Debeljkovic Dr., S. B. Stojanovic, and A. M. Jovanovic, "Further results on finite timeand practical stability of linear continuous time delay systems," FME Transactions, vol. 41, no. 3, pp. 241-249, 2013.

[5] L. Weiss and E. F. Infante, "Finite time stability under perturbing forces and on product spaces," IEEE Transactions on Automatic Control, vol. 12, pp. 54-59, 1967.

[6] F. Amato, M. Ariola, and C. Cosentino, "Finite-time stabilization via dynamic output feedback," Automatica, vol. 42, no. 2, pp. 337-342, 2006.

[7] F. Amato, M. Ariola, and P. Dorato, "Finite-time control of linear systems subject to parametric uncertainties and disturbances," Automatica, vol. 37, no. 9, pp. 1459-1463, 2001.

[8] F. Amato, R. Ambrosino, C. Cosentino, and G. De Tommasi, "Finite-time stabilization of impulsive dynamical linear systems," Nonlinear Analysis: Hybrid Systems, vol. 5, no. 1, pp. 89101, 2011.

[9] D. L. Debeljkovic, S. B. Stojanovic, and A. M. Jovanovic, "Finite time stability of continuous time delay systems: Lyapunovlike approach with Jensen's and Coppel's inequality," Acta Polytechnica Hungarica, vol. 10, no. 7, pp. 135-150, 2013.

[10] G. Garcia, S. Tarbouriech, and J. Bernussou, "Finite-time stabilization of linear time-varying continuous systems," IEEE Transactions on Automatic Control, vol. 54, no. 2, pp. 364-369, 2009.

[11] I. Karafyllis, "Finite-time global stabilizaton by means of timevarying distributed delay feedback," SIAM Journal on Control and Optimization, vol. 45, no. 1, pp. 320-342, 2006.

[12] M. P. Lazarević, D. L. Debeljković, Z. L. Nenadić, and S. A. Milinković, "Finite-time stability of delayed systems," IMA Journal of Mathematical Control and Information, vol. 17, no. 2, pp. 101-109, 2000.

[13] H. Liu and Y. Shen, " $H_{\infty}$ finite-time cont rol for switched linear systems with time-varying delay," Itelligent Control Automation, vol. 2, no. 3, pp. 203-213, 2011.

[14] E. Moulay, M. Dambrine, N. Yeganefar, and W. Perruquetti, "Finite-time stability and stabilization of time-delay systems," Systems \& Control Letters, vol. 57, no. 7, pp. 561-566, 2008.

[15] Y. Shen, H. Yu, and J. Jian, "Finite-time control for a class of discrete-time systems with time delay," in Proceedings of the 2nd International Symposium on Systems and Control in Aerospace and Astronautics, pp. 1-6, Shenzhen, China, 2008.

[16] S. B. Stojanović, D. L. Debeljković, and D. S. Antić, "Finite-time stability and stabilization of linear time-delay systems," Facta Universitatis. Series Automatic Control and Robotics, vol. 11, no. 1, pp. 25-36, 2012.

[17] M. Zhang, "Finite-time stability and stabilization of nonlinear quadratic systems with jumps," Mathematical Problems in Engineering, vol. 2014, Article ID 904607, 5 pages, 2014.
[18] G. Zong, R. Wang, W. X. Zheng, and L. Hou, "Finite-time stabilization for a class of switched time-delay systems under asynchronous switching," Applied Mathematics and Computation, vol. 219, no. 11, pp. 5757-5771, 2013.

[19] D. L. Debeljkovic, M. P. Lazarevic, S. A. Milinkovic, and M. B. Jovanovic, "Finite time stability analysis of linear time delay system: bellman-gronwall approach," in Proceedings of the IFAC Workshop on Linear Time Delay Systems, pp. 171-176, July 1998, Grenoble, France.

[20] F. Gao, Z. Yuan, and F. Yuan, "Finite-time control synthesis of networked control systems with time-varying delays," Advances in Information Sciences and Service Sciences, vol. 3, no. 7, pp. 1-9, 2011.

[21] J. Wang, J. Jian, and P. Yan, "Finite-time boundedness analysis of a class of neutral type neural networks with time delays," in Proceedings of the 6th International Symposium on Neural Networks on Advances in Neural Networks, pp. 395-404, Wuhan, China, May 2009.

[22] K. Gu, "An integral inequality in the stability problem of time-delay systems," in Proceedings of the 39th IEEE Confernce on Decision and Control, pp. 2805-2810, Sydney, Australia, December 2000.

[23] S. Boyd, L. El Ghaoui, E. Feron, and V. Balakrishnan, Linear Matrix Inequalities in System and Control Theory, vol. 15 of SIAM Studies in Applied Mathematics, Society for Industrial and Applied Mathematics (SIAM), Philadelphia, Pa, USA, 1994. 


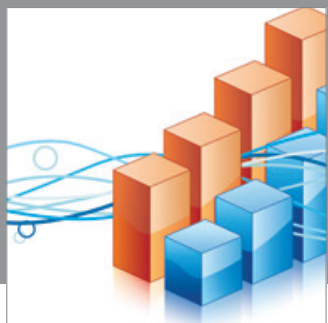

Advances in

Operations Research

mansans

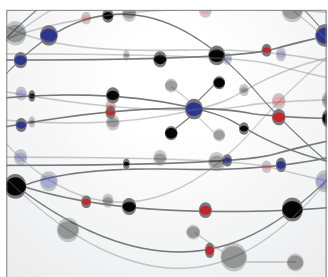

The Scientific World Journal
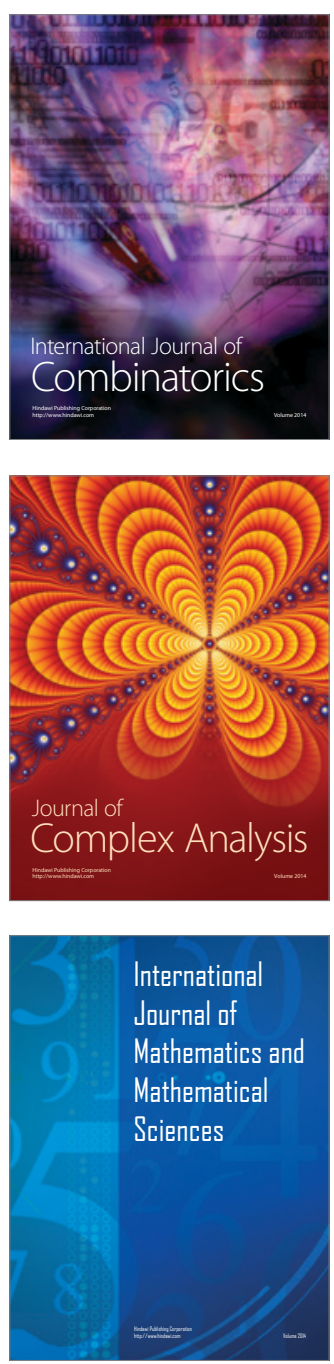
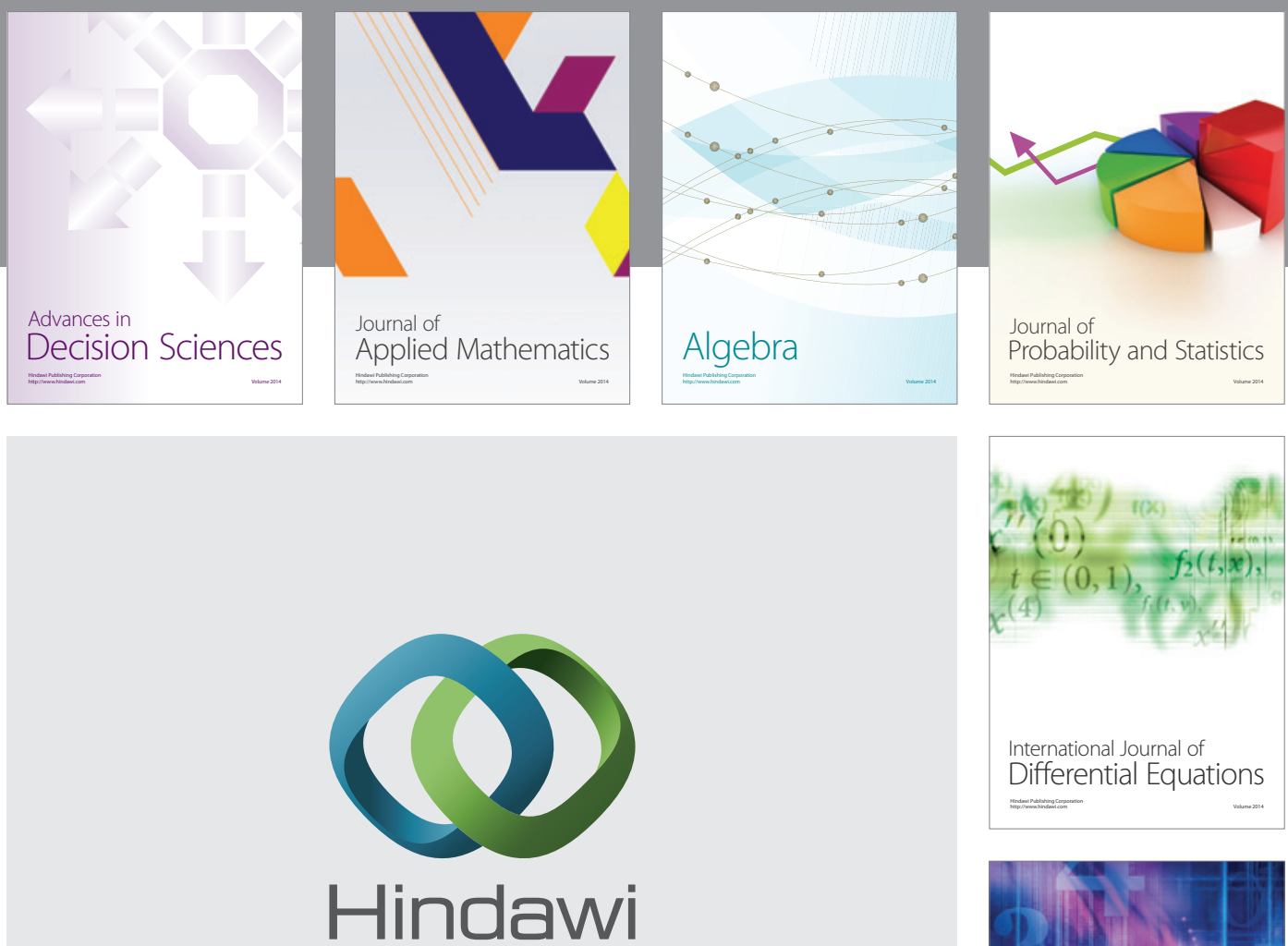

Submit your manuscripts at http://www.hindawi.com
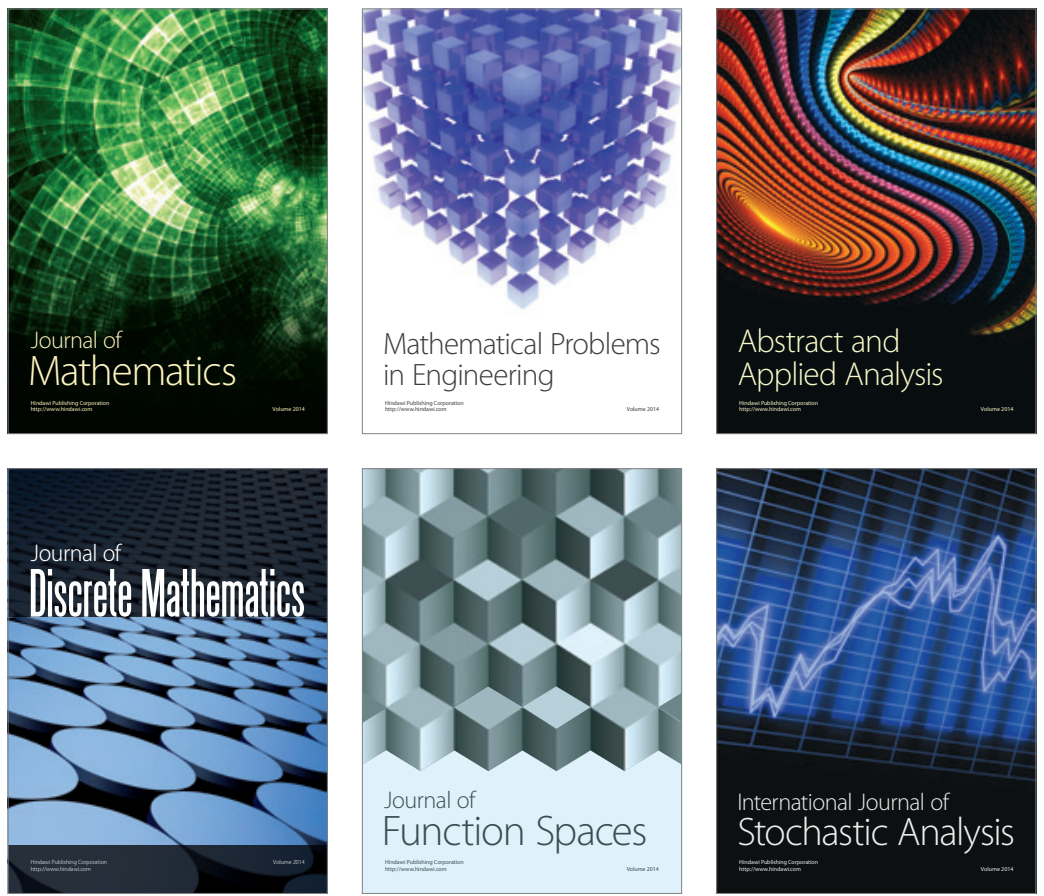

Journal of

Function Spaces

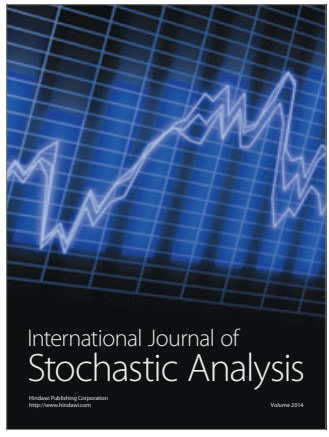

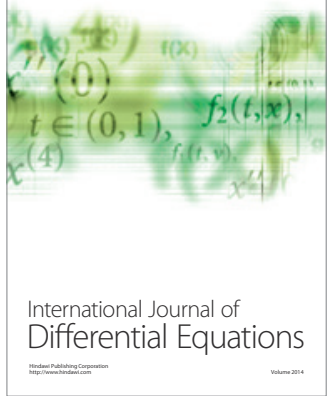
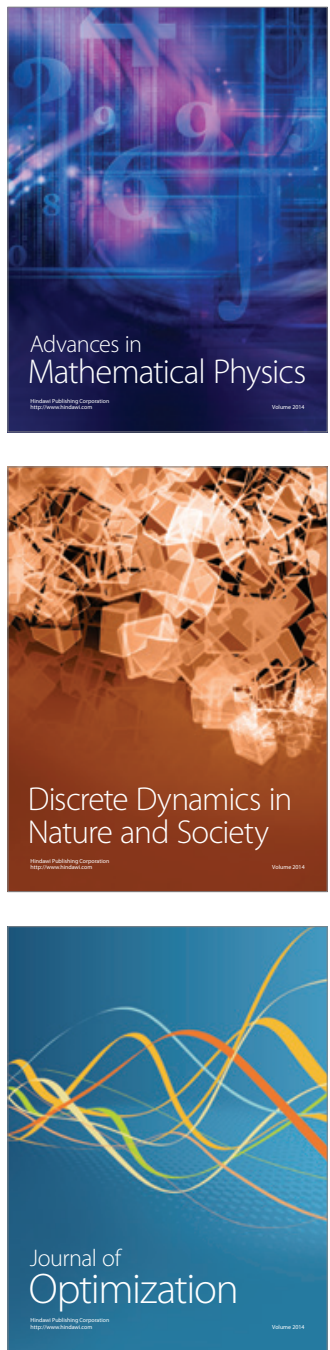